\title{
Inducción del trabajo de parto y resolución vaginal de gemelos unidos toraco-onfalópagos del segundo trimestre
}

\author{
Leoncio E. Jiménez-Alfonso*, Stephanie R. Tobón-Delgado, Jessica A. Mora-Galván y \\ Víctor H. Ramírez-Santes \\ Departamento de Ginecología y Obstetricia, Instituto Nacional de Perinatología, Isidro Espinosa de los Reyes (INPerIER). Ciudad de México, México
}

\section{Resumen}

Antecedentes: Los gemelos unidos o siameses son resultado de la división del disco embrionario más allá del día 13 luego de la fecundación. Estos casos, a pesar de su baja incidencia, tienen relevancia por las dificultades que representan para su atención. Presentación de casos: Caso 1: embarazo de 25.5 SDG gemelar monocorial monoamniótico, con fetos toraco-onfalópagos. Se realizó interrupción del embarazo por mal pronóstico fetal con esquema de mifepristona-misoprostol y uso de bomba de oxitocina. Parto por vía vaginal, sin complicaciones. Caso 2: embarazo de 19 SDG, gemelar monocorial monoamniótico, con fetos toraco-onfalópagos. Se interrumpió el embarazo por mal pronóstico fetal con esquema de mifepristona-misoprostol. Parto por vía vaginal, sin complicaciones. Conclusiones: Ante un embarazo con gemelos unidos en el segundo trimestre, que requieren interrupción del embarazo por mal pronóstico fetal, el parto vaginal, con una adecuada maduración cervical y conducción del trabajo de parto es una opción segura y evita los riesgos quirúrgico relacionados con una histerotomía.

Palabras clave: Embarazo gemelar. Gemelos unidos. Toraco-onfalópagos. Parto vaginal.

\section{Induction of labour and vaginal delivery of thoraco-omnphalopagus conjoined twins in the second quarter}

\section{Abstract}

Background: Conjoined or Siamese twins are the result of the division of the embryonic disc beyond $13^{\text {th }}$ day after fertilization. In these cases, despite having a low incidence, are relevant because of the difficulties they face in handling them. Presentation of cases: Case 1: Pregnancy of 25.5 weeks, monoamniotic monochorionic, thoraco-omphalopagus conjoined twins. Pregnancy was interrupted due to poor fetal prognosis with scheme: mifepristone - misoprostol and use of oxytocin pump. Vaginal delivery, without complications. Case 2: Pregnancy 19 of 25.5 weeks, monoamniotic monochorionic, thoraco-omphalopagus conjoined twins. Interruption of pregnancy was performed due to poor fetal prognosis with scheme: mifepristone - misoprostol. Vaginal delivery, without complications. Conclusions: Faced with a pregnancy with twins joined in the second trimester, which warrant interruption of pregnancy due to poor fetal prognosis, vaginal delivery, with adequate cervical ripening and labor conduction is a safe option, thus avoiding the surgical risks associated with a hysterotomy

Key words: Twin pregnancy. Conjoined twins. Thoraco-omphalopagus. Vaginal delivery.

\section{Correspondencia:}

*Leoncio E. Jiménez-Alfonso

E-mail: leo_13_97@ hotmail.com
Disponible en internet: 12-11-2021 Perinatol Reprod Hum. 2020;34(1):34-38 www.perinatologia.mx 0187-5337/C 2021. Instituto Nacional de Perinatología Isidro Espinosa de los Reyes. Publicado por Permanyer. Este es un artículo open access bajo la licencia CC BY-NC-ND (http://creativecommons.org/licenses/by-nc-nd/4.0/) 


\section{Introducción}

En teoría, los embarazo gemelares monocigotos ocurren por una división del cigoto (hasta el momento se desconocen los factores que la producen), lo que da lugar a embarazos bicoriales-biamnióticos si la división ocurre entre el primer y tercer días, embarazos monocoriales-biamnióticos si sucede entre los días cuarto a octavo, y monocoriales- monoamnióticos si se presenta entre los días octavo a decimotercero. Los gemelos unidos o siameses son resultado de la división del disco embrionario más allá del día 13 posterior a la fecundación ${ }^{1-3}$. La prevalencia mundial de gemelos unidos es de 1 caso por cada 100 embarazos de gemelos monocigotos, 1 de cada 50,000-100,000 embarazos espontáneos 0 en 1 de cada 200,000 nacidos vivos; en 2011 se notificaron los países con mayor prevalencia: India (1:2,800), Uganda $(1: 4,242)$ y Taiwán $(1: 6,500)$; España y Estados Unidos comunicaron la menor prevalencia $(1: 151,500$ y 1:200,000, respectivamente) $)^{3-5}$.

El pronóstico fetal es casi siempre malo debido a la presencia de anomalías congénitas graves que a menudo impiden la supervivencia de uno o ambos gemelos, incluso si se realiza la separación quirúrgica. En consecuencia, en algunos casos se decide la interrupción del embarazo; habitualmente la histerotomía es una opción para la resolución, que se relaciona con riesgos quirúrgicos y anestésicos. Se presentan dos casos de interrupción del embarazo por vía vaginal de fetos unidos toraco-onfalópagos del segundo trimestre que evitaron la histerotomía en ambas pacientes, lo que demostró que en situaciones seleccionadas es seguro efectuar una resolución vía vaginal.

\section{Casos clínicos}

\section{Caso 1}

Paciente de 26 años, gestaciones 2, cesárea 1, con embarazo gemelar monocorial monoamniótico, logrado de forma espontánea, de 25.5 semanas de gestación por ultrasonido. Los servicios de medicina maternofetal y cardiología fetal del INPerIER la valoraron, con informe de embarazo gemelar monocorial monoamniótico, fetos toraco-onfalópagos. En tórax: gemelo A con levocardia, cuatro cavidades, con CIV de $6 \mathrm{~mm}$ (sin arteria pulmonar) con única vía de salida ventricular; gemelo $\mathrm{B}$ con dextrocardia, cuatro cavidades cardiacas, con salida de aorta y arteria pulmonar; ambos gemelos compartían saco pericárdico.

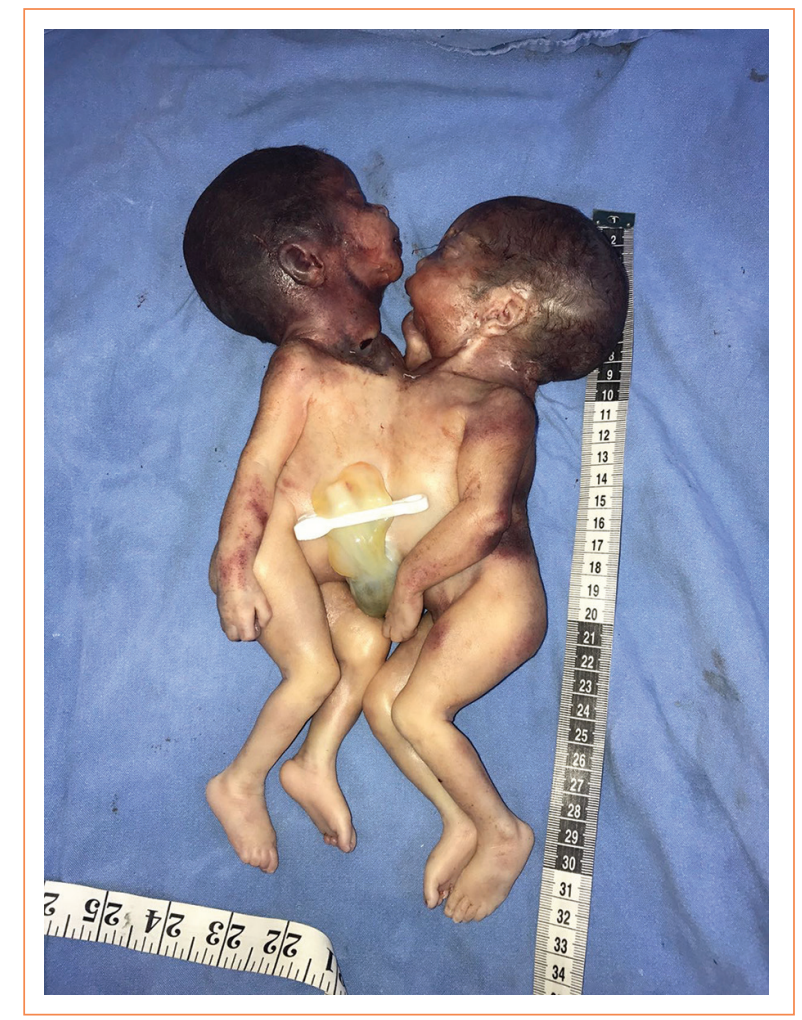

Figura 1. Gemelos toraco-onfalópagos del caso 1 obtenidos por parto vaginal. Ballard calculado de 26 semanas.

Medición torácica conjunta con diámetro de $10.14 \mathrm{~cm}$. En el abdomen se identificaron dos estómagos, dos vejigas, y compartían el área hepática, cordón umbilical con dos venas y tres arterias. Se estableció un mal pronóstico fetal, por lo que la paciente solicitó la interrupción del embarazo. De acuerdo con la presentación cefálica, diámetro conjunto y pelvis materna adecuada, se decidió una resolución vaginal. Se inició esquema de mifepristona (200 mg VO en dosis única) y misoprostol ( $200 \mu \mathrm{g}$ vaginal) a las $24 \mathrm{~h}$ posteriores y revaloraciones cada 6 h; se alcanzó una dosis máxima de misoprostol de 1,200 $\mu \mathrm{g}$, con modificaciones cervicales mínimas, por lo que se decidió envió a la unidad tocoquirúrgica (UTQ), donde se realizó analgesia obstétrica (bloqueo peridural) y se inició bomba de oxitocina (50 UI de oxitocina IV para 3 h). Una hora después pasó a sala de expulsión y atención del parto:

- Se dirigió el nacimiento del polo cefálico del gemelo A y luego del gemelo B, con maniobra de Ritgen modificada para su extracción (Fig. 1 y Tabla 1).

- Alumbramiento sin complicaciones, complementado con aspiración intrauterina. 
Tabla 1. Hallazgos del caso 1

\section{Neonatales (véase Fig. 1)}

Descripción: gemelos toraco-onfalópagos femeninos. Sin vitalidad.

Somatometría: peso total, 1,400 g; talla gemelo A, $31.5 \mathrm{~cm}$; gemelo B, $32.5 \mathrm{~cm}$.

\section{Maternos}

Lesiones cervicales o vaginales: no.

Desgarros perineales: 2 do grado (reparado).

Atonía uterina/hemorragia: $\mathrm{no} / 300 \mathrm{ml}$.

Se informan los hallazgos neonatales y maternos posteriores a la atención del parto del caso 1

El destino fetal fue el servicio de patología y no se autorizó la necropsia. La paciente evolucionó estable y egresó al siguiente día.

\section{Caso 2}

Paciente de 24 años, gestaciones 2, cesárea 1, referida al INPerlER con diagnóstico de embarazo gemelar monocorial monoamniótico de 19 semanas por ultrasonido con gemelos unidos toraco-onfalópagos. Al ingreso la valoraron los servicios de medicina maternofetal y cardiología fetal en los se concluyó: embarazo gemelar monocorial monoamniótico de 19 semanas por fetometría promedio; gemelo A: peso fetal calculado de $246 \pm 36 \mathrm{~g}$; gemelo B: peso fetal calculado de $260 \pm 38 \mathrm{~g}$. Ambos gemelos se encontraban unidos a nivel del tórax y compartían corazón, esternón, diafragma e hígado, con pulmones al parecer independientes; se observaron dos cámaras gástricas; columnas vertebrales en ambos productos de características habituales, ambas vejigas presentes; en la entrada del cordón umbilical ambos estaban fusionados, con pelvis separadas. El servicio de cardiología fetal efectuó una valoración y reconoció corazón único compartido por ambos fetos, lo que imposibilitaba la separación al nacimiento y sugería mal pronóstico para la vida. Ante los hallazgos notificados, los padres solicitaron la interrupción del embarazo.

Se inició la interrupción con esquema de mifepristona (200 mg en dosis única) y 24 h después se aplicó la primera dosis de misoprostol (200 $\mu \mathrm{g}$ por vía vaginal), con revaloraciones cada $6 \mathrm{~h}$ hasta alcanzar una dosis total de $400 \mu \mathrm{g}$ con modificaciones cervicales favorables. Se la envió a UTQ, donde se administró analgesia obstétrica, se confirmó la presentación

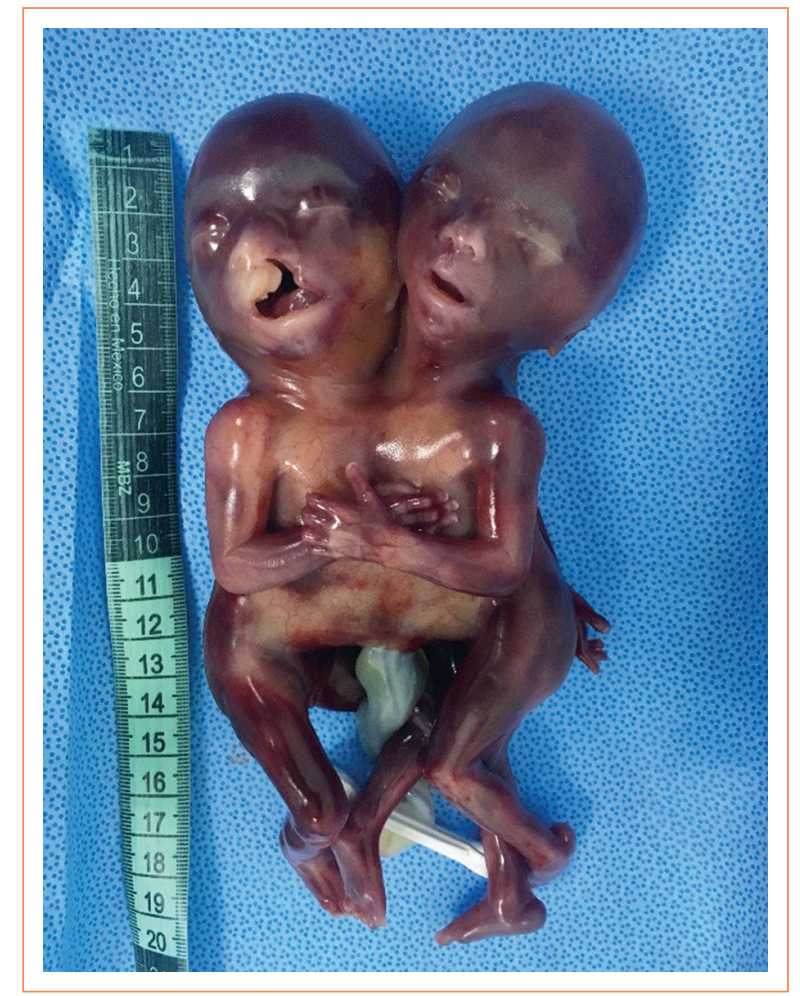

Figura 2. Gemelos toraco-onfalópagos del caso 2 obtenidos por parto vaginal. Ballard calculado de 20 semanas.

pélvica y dos horas después pasó a sala de expulsión para la atención del parto pélvico:

- Se realizó la maniobra de Bracht con liberación de ambas pelvis, luego se liberaron los hombros con maniobra de Rojas y se extrajeron ambos gemelos (Fig. 2 y Tabla 2).

- Alumbramiento sin complicaciones que se complementó con revisión de la cavidad (curetaje).

Lo fetos se destinaron al departamento de patología y no se autorizó la necropsia. La paciente evolucionó favorablemente y egresó a las $24 \mathrm{~h}$.

\section{Discusión}

En 1996, la doctora Rowena Spencer describió una clasificación para los gemelos unidos con base en el punto de unión (Tabla 3$)^{6}$ : debido al incremento del uso de ultrasonido, el diagnóstico de gemelos unidos puede efectuarse durante el tamizaje del primer trimestre; muchos informes de caso describen este diagnóstico antes de la semana 15 de gestación?

La conducta terapéutica de los gemelos unidos se basa en gran medida en datos de informes de casos, series pequeñas de casos y opiniones de expertos. Cuando los 
Tabla 2. Hallazgos del caso 2

Neonatales (véase Fig. 2)

Descripción: gemelos toraco-onfalópagos femeninos. Sin vitalidad.

Somatometría: peso total, $406 \mathrm{~g}$; talla gemelo A, $19.5 \mathrm{~cm}$ (labio y paladar hendido); gemelo $\mathrm{B}, 20 \mathrm{~cm}$.

\section{Maternos}

Lesiones cervicales o vaginales: no.

Desgarros perineales: no.

Atonía uterina/hemorragia: no/100 ml.

Se informan los hallazgos neonatales y maternos posteriores a la atención del parto del caso 2

Tabla 3. Clasificación de Spencer para los gemelos unidos8

\footnotetext{
Unión ventral

Cefalópagos: unidos que comparten el encéfalo.

Toracópagos: Unidos por tórax a ombligo; comparten

esternón, diafragma, hígado, corazón, aunque sea sólo por un vaso interauricular.

Onfalópagos: unidos a nivel del ombligo; comparten hígado, íleon y colon.

Isquiópagos: unidos por cadera, comparten tubo digestivo distal y tracto genitourinario.

Unión lateral

Parápagos: unión lateral, comparten abdomen inferior, tracto genitourinario y anorrectales.

Unión dorsal

Craneópagos: unidos por el cráneo, comparten cráneo, meninges y senos venosos.

Raquiópagos: unidos por la columna.

Pigópagos: unidos por el sacro, comparten sacro, cóccix, huesos pélvicos, ano y recto.
}

padres deciden continuar con el embarazo, se recomienda un seguimiento ecográfico por un especialista en medicina maternofetal, pero existe un riesgo significativo de muerte fetal aproximado del $40 \%$, así como riesgo mayor de parto de pretérmino ${ }^{8,9}$. Se ha informado que sólo $40 \%$ a $60 \%$ de los gemelos unidos nacen vivos y de éstos más del $50 \%$ mueren en el período neonatal ${ }^{10}$.

Debido al mal pronóstico de los gemelos unidos, la mayoría de los padres considera la interrupción electiva del embarazo. La terminación temprana (primer trimestre) reduce al mínimo el riesgo de morbilidad materna. En el primer o segundo trimestres (hasta las 20 semanas), la terminación quirúrgica por dilatación y evacuación es una opción ${ }^{8,10}$.

Para pacientes en el segundo trimestre tardío (>25 semanas) o tercer trimestre se recomienda la resolución por vía abdominal (histerotomía-cesárea), ya que de esta manera se elimina el riesgo de parto obstruido y disminuye el riesgo de ruptura uterina y traumatismo en el tracto genital de la madre. En estos casos, la incisión uterina óptima depende de factores específicos del paciente, incluidos la edad gestacional, el tipo de inserción y el pronóstico neonatal. Las incisiones abdominales y uterinas deben ser lo suficientemente grandes para liberar a los gemelos, lo cual exige casi siempre una incisión uterina común. Se debe considerar que la resolución abdominal conlleva riesgos inmediatos (riesgos quirúrgicos inherentes) y riesgos a largo plazo (incisión uterina típica, placenta previa y acretismo placentario en gestaciones posteriores) ${ }^{8,10}$.

Por otra parte, se han notificado casos de gemelos unidos $>25$ semanas con resolución por parto vaginal con éxito. En 2009 se publicó un informe de tres casos de gemelos unidos $>28$ semanas obtenidos por vía vaginal sin complicaciones graves. En otro estudio en 2011, en una serie de más de 30 casos, la resolución por vía vaginal de embarazo de gemelos unidos fue una intervención segura en pacientes con $>25$ semanas $^{8,11}$.

Se presentan dos caso de un embarazo gemelar monocorial, monoamniótico con diagnóstico de gemelos toraco-onfalópagos, ambos en el segundo trimestre de gestación, valorados por los servicios de obstetricia, medicina maternofetal y cardiología fetal; sin embargo, dada la complejidad y mal pronóstico para la vida de los gemelos, se determinó la resolución del embarazo en ambos casos. Antes de la interrupción, el servicio de genética le explicó a las pacientes que las causas del padecimiento no eran de origen hereditario y no eran necesarios estudios complementarios. Asimismo, el servicio de psicología orientó a la pareja en el afrontamiento de las decisiones tomadas.

En ambas pacientes se consideraron las condiciones obstétricas, con base en lo cual se decidió la resolución por vía vaginal mediante conducción inducida del trabajo de parto de pretérmino, bajo el esquema combinado de mifepristona-misoprostol. Para el caso 1 fue necesario el uso de bomba de oxitocina para favorecer una rápida conducción del trabajo de parto de pretérmino. Al final, el adecuado conocimiento y ejecución correcta de las maniobras obstétricas en la atención del parto produjeron una resolución vaginal exitosa con mínimo traumatismo del canal de parto, sin manipulación fetal excesiva ni complicaciones.

\section{Conclusiones}

La atención de los gemelos unidos depende de la edad gestacional, la esperanza de vida y el pronóstico. En caso de resolución del embarazo, la vía vaginal 
debe reservarse para fetos pequeños no viables y la histerotomía-cesárea para fetos grandes o con mayor expectativa de vida y pronóstico.

Ante un embarazo con gemelos unidos en el segundo trimestre, que requieran interrupción del embarazo por mal pronóstico fetal, el parto vaginal, con una adecuada maduración cervical y conducción del trabajo de parto, es una opción segura y evita de esta forma los riesgos que implica una laparotomía e histerotomía. Sin embargo, los autores recomiendan que siempre se tengan al alcance el equipo médico y los recursos materiales para atender una distocia fetal o practicar una laparotomía urgente.

\section{Conflicto de intereses}

Los autores, médicos residentes y adscritos del INPer IER, declaran que actuaron bajo los más estrictos principios de la ética profesional y no existe ninguna situación de conflicto de intereses real, potencial o evidente.

\section{Financiamiento}

Esta investigación no se realizó bajo ningún financiamiento externo. Los gastos que la misma implicó fueron cubiertos por los autores.

\section{Responsabilidades éticas}

Protección de personas y animales. Los autores declaran que para esta investigación no se han realizado experimentos en seres humanos ni en animales.

Confidencialidad de los datos. Los autores declaran que han seguido los protocolos de su centro de trabajo sobre la publicación de datos de pacientes.

Derecho a la privacidad y consentimiento informado. Los autores han obtenido el consentimiento informado de los pacientes y/o sujetos referidos en el artículo. Este documento obra en poder del autor de correspondencia.

\section{Bibliografía}

1. Mcnamara HC, Kane SC, Craig JM, Short RV, Umstad MP. A review of the mechanisms and evidence for typical and atypical twinning. Am J Obst Gynecol. 2015. doi: 10.1016/j.ajog.2015.10.930.

2. Mutchinick OM, Luna-Muñoz L, Amar E. Conjoined twins: a worldwide collaborative epidemiological study of the International Clearinghouse for Birth Defects Surveillance and Research. Am J Med Genet C Semin Med Genet. 2011:157C:274.

3. Brizot L. Conjoined twins pregnancies: experience with 36 cases from a single center. Prenat Diagn. 2011;31:1120-1125.

4. López-Márquez A. Gemelos toracópagos: estudio post morten. Rev Med Hosp Gen Mex. 2003;66(1):37-42.

5. González-Cortés BGI, Hernández-Valencia M. Siameses toracópagos: presentación de caso y revisión de la literatura. Perinatol Reprod Hum. 2015;29(3):130-133

6. Spencer R. Anatomic description of conjoined twins: a plea for standarized terminology. K Ped Surg. 1996;31(7):941-944.

7. Chih-Ping $\mathrm{C}$, Chin-Yuan H, Jun-Wei S. Conjoined twins detected in the first trimester: a review. Taiwanese J Obstet Gynecol. 2011;50:424e431.

8. O'Brien P, Nugent M, Khalil A. Prenatal diagnosis and obstetric management. Semin Pediatr Surg. 2015;24:203.

9. Mackenzie TC, Crombleholme TM, Johnson MP. The natural history of prenatally diagnosed conjoined twins. J Pediatr Surg. 2002;37:303.

10. Mitchell T, Cheng E, Jolley J. Labor induction of conjoined twins. Obstet Gynecol. 2014;123:469-72.

11. Tug N, Sahinoglu Z, Cam C, Eren S. Route of delivery of thoracoomphalopagus twins: analysis of three cases. Arch Gynecol Obstet. 2009;280:815. 